\section{Resolución de Problemas Previo a Instrucción, aplicado al aprendizaje online de Modelos de Atención Selectiva}

\section{Susana Carnero-Sierra y Eduardo González-Cabañes}

Universidad de Oviedo

\section{Resumen}

Se aplicó el enfoque de Resolución de Problemas Previo a la Instrucción (RP-I) a la enseñanza de modelos atencionales en la asignatura de Psicología de la Atención del grado de Psicología. El enfoque respondió a la necesidad de adaptación online durante el confinamiento generado por la crisis sanitaria. Este abordaje pedagógico hace al alumnado partícipe del proceso de aprendizaje, especialmente complicado dentro de la enseñanza de la atención selectiva desde el enfoque experimental. Desde el campus virtual de la asignatura y antes de la explicación de modelos atencionales, se planteó al alumnado la resolución de un problema en que debían confeccionar sus propios modelos atencionales. Al finalizar esta actividad, se pidió a los alumnos que informaran de las emociones que habían experimentado durante la misma. También se evaluó su nivel de participación y su desempeño en la actividad. Los alumnos mostraron una muy alta participación en el problema planteado, así como una riqueza y variedad de soluciones del problema. La mayoría mostraron un empleo maduro de la terminología específica. El enfoque RP-I contribuyó al aprendizaje de la asignatura y promovió una alta participación e implicación en el aprendizaje, enriqueciendo el formato de docencia no presencial.

Palabras clave: Métodos docentes, universitarios, respuesta emocional, resolución de problemas, habilidades de pensamiento, fallo productivo.
Problem-solving before Instruction applied to online learning of Selective Attention Models

\section{Abstract}

Introduction. The Problem-solving before Instruction Approach (PS-I) was applied to the teaching of attentional models in the subject of Psychology of Attention. The approach responded to the need of adapting to the online classes during the confinement generated by the health crisis. This pedagogical approach makes students participate in the learning process, especially complicated within the teaching of selective attention models. Methodology. Before the instruction about models of attention, from the moodle of the course students were asked to solve a problem in which they had to invent their own models of attention. At the end of this activity, students were asked to report the emotions they had experienced during it. Their level of participation and their performance in the activity were also evaluated. Results. Students showed a remarkably high participation, as well as quality and variety in the solutions provided to the problem. Most showed a mature use of specific terminology. Conclusions. The PS-I promoted a good understanding of the subject and a high involvement of students in the learning process, enriching the format of non-face-to-face teaching.

Key words: Teaching methods, higher education, emotional response, problem solving, thinking skills, productive failure.

\section{Introducción}

Los docentes a menudo nos preguntamos si, antes de explicar un concepto importante, vale la pena que los alumnos resuelvan de manera previa problemas sobre ello. En otras palabras, que inductivamente se enfrenten a una situación no resuelta antes de enfrentarse a las soluciones normativas que les facilitamos en las explicaciones de las clases teóricas. Por un lado, es una inversión de tiempo en la que los alumnos quizá se exijan con frustración, o atiendan a aspectos irrelevantes para los objetivos docentes marcados (Clark, Kirschner y Sweller, 2012). Por otro lado, puede contribuir a que los alumnos reflexionen críticamente a través de varios mecanismos. Los estudiantes pueden hacerse más conscientes de las lagunas de sus conocimientos (Loibl y Rummel, 2014); sentirse más motivados por el rol creativo

\title{
MAGISTER
}

Vol. 32. Núm. I: (2020). Sección extraordinaria 
y la oportunidad de servirse de conocimientos personales (Belenky y Nokes-Malach, 2012); y activar conocimientos previos que pueden facilitar la asimilación de contenidos vistos más adelante (Kapur, 2014).

La literatura científica ha mostrado resultados divergentes sobre la eficacia del enfoque de resolución de problemas previo a la instrucción (Loibl, Roll y Rummel, 2017), lo que puede depender de los contenidos que se quieran enseñar. Específicamente, este tipo estrategias pedagógicas de amplia aplicación educativa, apenas se ha usado en la enseñanza de modelos experimentales sobre procesos psicológicos básicos. Dentro del currículo académico en psicología, son varios los retos que el alumno tiene que afrontar como inesperados en cuanto a intersección con las áreas más cercanas a la metodología experimental: medida, variables, estadística y la lógica y terminología de diseños de corte cognitivo y modelos empíricos. De entre estas materias, la Psicología de la Atención destaca, además, por su reciente incorporación y aparición independiente como asignatura obligatoria, desde la implantación del sistema de grados en nuestro país. Es por esto por lo que la docencia de determinados contenidos dentro de esta asignatura es, a veces, difícil y árida de plantear, añadido a la desconfianza del alumno ante términos abstractos sobre modelos y predicciones experimentales. A este respecto, se observa que los alumnos se sienten sobrecargados ante la complejidad de las explicaciones y acompañamiento de datos experimentales, lo que los lleva a mostrar baja motivación para seguir aprendiendo. Esta desconexión se hace patente en las evaluaciones y en la capacidad crítica del alumnado al expresar y resolver dudas, mostrando dificultades para aplicar los modelos atencionales a situaciones de la vida real y para identificar las diferencias y similitudes entre unos modelos y otros. Esta cuestión, se suma a la escasez de manuales en castellano, unificados, críticos, completos y con perfil didáctico, dada su reciente implantación independiente en el grado, y a la situación de la asignatura en el primer curso, donde las explicaciones se trabajan sobre una base aún incompleta de conceptos propios de la psicología. Además, la propia dificultad del estudio y conceptualización del fenómeno atencional genera una complejidad entretejida con los factores previamente expuestos, que permite un caldo de cultivo especialmente beneficioso para la implantación de una experiencia a través del Enfoque de Resolución de Problemas Previo a la Instrucción (RP-I). A través de la resolución de un problema previo, se puede favorecer su motivación y familiarización con los contenidos (Belenky y Nokes-Malach, 2012), lo que puede ser crucial para que no se genere una indefensión conceptual desde las primeras explicaciones.

Enriqueciendo esta serie de complejidades, la aplicación de esta experiencia se enmarcó en la necesidad de adaptar la docencia al formato online no presencial, debido a la crisis sanitaria de la Covid-19. Esta situación marcó, aún más si cabe, la necesidad de incorporar estrategias que den autonomía a los alumnos y de disponer de estrategias de observación de las emociones experimentadas a la hora de aprender, debido a la situación de incertidumbre general y particular de la adaptación súbita al entorno digital. Por todo ello, el objetivo la experiencia fue aplicar este enfoque (RP-I) a la enseñanza de modelos atencionales y evaluar su eficacia sobre una parte del temario de la asignatura de Psicología de la Atención destinada a fijar conceptos sobre los primeros modelos sobre atención selectiva de los años 50-70 del siglo XX.

Antes de explicar varios de los modelos atencionales más aceptados científicamente, se dio la oportunidad al alumnado de que inventaran sus propios modelos atencionales bajo unas directrices y desde un punto de partida. Los alumnos y alumnas partieron de un ejemplo de un modelo atencional básico y de resultados experimentales que debían ser integrados en los modelos que construyeran. Una vez que entregaron sus respuestas, se realizó la explicación de la teoría que contaba cómo han sido en la realidad los modelos atencionales aceptados, así como se proporcionó un feedback con el resumen de los modelos propuestos y su grado de viabilidad. Al acabar estas dos actividades se evaluaron las emociones que experimentaron, su satisfacción con la nueva metodología y la calidad de las respuestas que proporcionó el alumnado.

\section{Objetivos/Hipótesis}

El objetivo general de la experiencia fue aplicar el enfoque RP-I en el aprendizaje de modelos de Atención Selectiva, como estrategia que puede ser efectiva para fomentar la motivación y para adaptar la enseñanza al aprendizaje virtual sobrevenido por el confinamiento derivado de la actual pandemia. Específicamente se planteó realizar un seguimiento de las emociones experimentadas al enfrentarse a la actividad, así como una observación de si se había producido la integración de

\section{MAGISTER}

Vol. 32. Núm. I: (2020). Sección extraordinaria 
terminología propia de la construcción, expresión y discusión de modelos de corte cognitivo.

Se esperaron puntuaciones altas en el desempeño del problema, aunque ello llevara asociado la experimentación de emociones negativas como la frustración y la confusión. Lejos de ser contradictorio, encontrar parámetros en ambos polos de emociones sería índice de la solidez de aprendizajes construidos sobre retos y dificultades, pero asumibles y superables. Además, se consideró que la evaluación y análisis cualitativo de las respuestas del alumnado reflejaría un dominio maduro de los términos propios de la Atención Selectiva y una comprensión de la aplicabilidad de los modelos a situaciones de la vida cotidiana, que aseguraría una comprensión de los datos experimentales explicados en las clases previas proporcionadas a través del campus virtual de la asignatura, reforzando y enriqueciendo de manera profunda el enfoque no presencial.

\section{Método/ Metodología}

El proyecto se llevó a cabo en la asignatura de Psicología de la Atención en $1^{\circ}$ Psicología. Se implementaron los materiales educativos necesarios para iniciar el proceso de la experiencia, explicando un primer modelo atencional de características cognitivas (Broadbent, 1958) mediante audio-clases colgadas en el campus y videoconferencias de discusión y dudas en tiempo real con el alumnado. Se explicaron a su vez los experimentos que apoyan y también los que ponen problemas a dicho modelo. A continuación, como problema de invención previo a la instrucción, se abrió una tarea en el campus virtual a través de un documento con la descripción que se puede consultar en la Figura 1.

"A continuación, se muestra el modelo de Broadbent (1958) sobre cómo se filtra selectivamente la información, así como un resumen de los experimentos que hemos visto al respecto. Tu tarea es diseñar distintas propuestas de representaciones sobre cómo funciona la atención selectiva, y a sea modificando el diagrama de Broadbent o creando representaciones totalmente nuevas.

Es decir, crea tus nuevos modelos para que puedan dar cuenta del conjunto de los resultados experimentales que hemos visto. Puedes añadir nuevas estructuras y/o nuevas relaciones (flechas) entre estructuras, cambios de orden en la secuencia de procesamiento, nuevos supuestos...

Figura 1. Instrucciones proporcionadas al alumnado para orientar la tarea de invención previa a la instrucción.
Fueron informados los 125 alumnos matriculados de la voluntariedad de la participación, que suponía no obstante la suma de una pequeña puntuación proporcional a la calidad de su ejecución. Fueron informados que el total de puntuación se obtendría con un balance entre viabilidad de las propuestas, número de modelos generados, madurez de expresión y originalidad. Los estudiantes podían construir sus modelos a partir de transformaciones de este modelo de referencia, o creando modelos completamente nuevos. Para fomentar que los estudiantes reflexionaran más sobre el tema y activaran una mayor cantidad de conocimientos previos, se les dio la oportunidad de crear tantas soluciones como fueran capaces.

Una sesión de feedback de clase expositiva fue colgada en diferido en el campus al finalizar el plazo de entrega, como así también estuvo disponible una presentación power point con un resumen de sus aportaciones de modo anónimo. Al finalizar el problema de invención, los alumnos reportaron las emociones que experimentaron durante el aprendizaje. Al final de las clases expositivas también tuvieron la oportunidad de contestar voluntariamente a cuatro ítems cuya respuesta debían dar en escala tipo Likert ( $1=$ Nada, $2=$ Poco, $3=$ =Algo, 4=Bastante, $5=$ =Mucho) para evaluar la satisfacción con la metodología utilizada, importancia de los contenidos, transferencia de lo aprendido e interés general que experimentaron al realizar la tarea.

Para evaluar las emociones que experimentaron los alumnos durante el aprendizaje, se utilizó una adaptación al español de la escala Epistemic Related Emotions Questionnaire (Pekrun, Vogl, Muis, y Sinatra, 2017), elaborada expresamente para la experiencia. Este cuestionario está compuesto por siete escalas que se corresponden con siete emociones epistémicas, es decir, emociones que pueden tener implicaciones en el aprendizaje: sorpresa, curiosidad, disfrute, confusión, ansiedad, frustración y aburrimiento. Cada una de estas subescalas estaba compuesta por tres ítems que representaban la emoción. Los estudiantes respondían indicando el grado de intensidad con que experimentaron dichas emociones a través de una escala tipo Likert $(1=$ Nada, $2=$ Muy Poco, $3=$ Moderadamente, $4=$ Mucho, $5=$ Muchísimo). La fiabilidad interna encontrada en esta muestra fue bastante alta para todas las escalas: sorpresa $(\alpha=.763)$, curiosidad $(\alpha=.734)$, disfrute $(\alpha=.865)$, confusión $(\alpha=.793)$, ansiedad $(\alpha=.690)$, frustración $(\alpha=.754)$ y aburrimiento $(\alpha=$ $.703)$.

\section{MAGISTER}

Vol. 32. Núm. I: (2020). Sección extraordinaria 


\section{Resultados}

Un total de 104 alumnos respondieron a la tarea, es decir el $83.2 \%$ del total de matriculados. De estos alumnos, 83 respondieron al cuestionario sobre emociones durante esta actividad. Sólo 14 estudiantes respondieron a las cuestiones sobre su interés en los contenidos y satisfacción con la metodología. Estos resultados sobre satisfacción indicaron que el 79\% de esta pequeña muestra informó sentirse bastante o muy satisfecho con su participación en el proyecto, mientras que ningún alumno eligió la opción nada satisfecho. El 57\% juzgó que la implantación del método contribuyó bastante o mucho a favorecer su aprendizaje. En el ítem sobre la importancia que concedían a los contenidos, el 79 \% respondió que les concedía bastante o mucha importancia a los contenidos trabajados. Sin embargo, para el ítem referido a en qué medida podrán utilizar lo aprendido en otros contextos, sólo el $28 \%$ lo juzgó como bastante y ninguno como mucho.

Respecto a las emociones, la Figura 2 muestra las medias y las desviaciones típicas para la intensidad con que los alumnos informaron experimentar cada una de las siete emociones durante el problema de invención. Podemos observar que los alumnos informaron de una mayor intensidad para las emociones de curiosidad y de confusión.

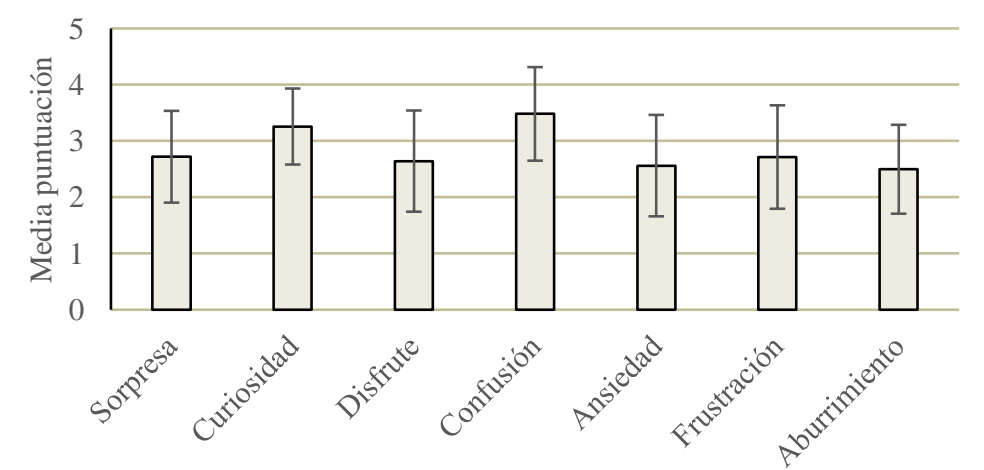

Figura 2. Descriptivos sobre las emociones experimentadas durante el problema de invención.
Respecto al desempeño los alumnos en el problema, se obtuvieron puntuaciones altas (Media $=7.77$, Desviación Típica $=1.66$ ), oscilando entre un rango de 4 a 10 dentro de una escala medida sobre 10. En concreto 2 alumnos suspendieron, 31 obtuvieron entre 5-6.5, 33 sacaron calificaciones de 7-8.5 y 38 obtuvieron entre 910. Es decir 71 alumnos (68\% del alumnado) sacaron notable o sobresaliente.

La evaluación de las propuestas en su perspectiva más conceptual arrojó los siguientes resultados de exploración cualitativa como feedback a la acción docente. La mayoría de los modelos propuestos adelantaban ideas que efectivamente protagonizaban las propias soluciones reales de los modelos que se explicarían a continuación. En primer lugar, el alumnado propuso diferentes jerarquías de mando en la cadena cognitiva para solucionar los datos empíricos que se les había requerido encajar en sus propuestas. Esto, a su vez, indicó una correcta y adecuada interiorización y manejo reflexivo de los conceptos de la propuesta cognitiva, la cual considera diversos fenómenos actuando de forma paralela y secuencial. En segundo lugar, aparecieron propuestas que no se limitaban a crear nuevos caminos o relaciones entre las estructuras del modelo que ya conocen, sino que propusieron nuevas estructuras, con su propia denominación y función dentro del sistema de procesamiento. En tercer lugar y también a un nivel general, surgió la discusión del sistema consciente/inconsciente incorporándose a muchas de las propuestas realizadas. Aunque el problema de la conciencia y su relación con la atención resultó problemático y en muchas propuestas no se encontraba rigurosamente bien empleado, los alumnos mostraron espontáneamente el planteamiento y la reflexión sobre este aspecto, complejo y de gran interés en la literatura de Psicología de la Atención. Por último y de manera puntual, se recabaron varias soluciones de alto interés y madurez, que conectaban con otros contenidos no explícitamente trabajados de manera previa: fenómenos de inhibición, habituación e irrelevancia estimular, selección como ventaja adaptativo-biológica, sistemas de control de la flexibilidad y relatividad de los filtros atencionales, así como articulación de sistemas heterogéneos para condiciones cognitivas diversas.

\section{MAGISTER}

Vol. 32. Núm. I: (2020). Sección extraordinaria 


\section{Discusión}

Como síntesis de la experiencia se puede concluir que se observó un aprendizaje de calidad, que sirvió para constatar la evolución del alumnado en ausencia de presencialidad, adaptándose a la perfección a las dificultades encontradas durante el confinamiento por la Covid-19. Las preguntas sobre satisfacción apuntan a buenos resultados de cara a consolidar esta tarea dentro de la metodología docente de esta asignatura. Sin embargo, la baja participación en estas preguntas, posiblemente debida a su coincidencia temporal con la mayor parte de la carga de tareas y preparación de exámenes de esta y el resto de las asignaturas, hace necesaria más observación al respecto y la repetición de sucesivas experiencias.

La calidad y creatividad mostrada en las respuestas de los alumnos al problema de invención es coherente con otros resultados de la literatura que muestran que el enfoque RP-I es efectivo para promover la comprensión de contenidos conocimientos (Loibl et al., 2017; Schwartz, Chase, Oppezzo y Chin, 2011), la consciencia sobre las propias necesidades de aprendizaje conocimientos (Loibl y Rummel, 2014) y la motivación de los estudiantes (Belenky y Nokes-Malach, 2012). De hecho, como novedad es importante señalar que las respuestas de los alumnos reflejaron objetivos propios de aprendizaje, distintos de los del docente o diferentes de los que el docente puede prever en los inicios del abordaje de los objetivos didácticos.

Los resultados refuerzan una conclusión extendida, pero difícilmente conseguida: los modelos atencionales requieren de reflexión para ser entendidos. Así mismo, los resultados sobre emociones también sugieren trabajo reflexivo por parte de los alumnos. Se encontraron altos grados de confusión, bajos valores de aburrimiento, pero considerables niveles de curiosidad. Este resultado es coherente con estudios recientes que sugieren que las emociones negativas como la confusión no tienen por qué ir necesariamente ligadas a procesos inefectivos de aprendizaje y que si se experimentan dentro de una situación percibida como desafiante y temporal pueden incluso favorecer procesos reflexivos de aprendizaje (D'Mello y Graesser, 2014; Di Leo, Muis, Singh y Psaradellis, 2019).

\section{MAGISTER}

Vol. 32. Núm. I: (2020). Sección extraordinaria
Aunque implique cierta frustración, el pensamiento reflexivo es crucial para poder aplicar los contenidos a lecciones que los alumnos se encuentran no sólo más adelante en la propia asignatura, sino en otros futuros problemas que participen de pensamiento científico. A este respecto es de destacar el resultado de las preguntas sobre el interés, donde el alumnado reconoce interesantes los contenidos, pero no los relaciona con su aplicabilidad en otros contextos. Futuros ejercicios, siguiendo la misma metodología por descubrimiento, debería diseñar nuevos ejercicios de invención donde el objetivo sea exprimir la reflexión sobre la aplicación de los modelos atencionales precisamente a otros contextos.

La aplicación de esta experiencia se enmarcó en el objetivo de mejorar las competencias transversales y extracurriculares del estudiantado, ya que el enfoque RP-I no sólo constituye una manera de preparar a los alumnos para el aprendizaje, sino también una oportunidad para que los alumnos se enfrenten a problemas novedosos y se vean ante el desafío de aportar soluciones creativas que requieran pensamiento crítico (Schwartz et al., 2011; Schwartz y Martin, 2004). Tanto el pensamiento crítico como la creatividad son capacidades que no pueden ser aprendidas por la pura memorización de reglas y su práctica es una condición fundamental para su desarrollo (Barth, Godemann, Rieckmann y Stoltenberg, 2007; Feriver, Olgan, Teksoz y Barth, 2019). Promover estas capacidades es especialmente importante si tenemos en cuenta que ambas habilidades pueden ser perfectamente facilitadas en el entorno virtual a través de la organización de tareas desde la lógica del RP-I, sin alta inversión tecnológica. Así mismo, la resolución de problemas previa a la instrucción también puede contribuir a hacer a los alumnos más resilientes y tolerantes a la frustración, pues se espera que fracasen durante la fase de resolución de problemas y que aprendan a aprender de estos errores en la fase de instrucción posterior (Simpson y Maltese, 2017).

\section{Conclusiones}

Los resultados sugieren que los alumnos experimentaron altos niveles de curiosidad durante la actividad y que activaron procesos de pensamiento crítico. La alta participación y la generalidad de uso de lenguaje técnico específico adecuado, constató la viabilidad del enfoque de Resolución de Problemas Previo a la Instrucción para promover el aprendizaje, reflexión y comprensión de modelos de 
Atención Selectiva, observándose una alta adecuación de la tarea, las instrucciones de esta y la secuencia del proceso de aprendizaje. Además, se validó la realización de esta experiencia a través del campus virtual como único modo de comunicación, ofreciendo una completa forma de evaluación de un aprendizaje complejo, sin recurrir al formato examen y aplicando formas pedagógicas reflexivas y dinámicas.

\section{Referencias}

Barth, M., Godemann, J., Rieckmann, M., \& Stoltenberg, U. (2007). Developing Key Competencies for Sustainable Development in Higher Education. International Journal of Sustainability in Higher Education, 8, 416-430. doi:10.1108/14676370710823582

Belenky, D. M., \& Nokes-Malach, T. J. (2012). Motivation and Transfer: The Role of Mastery-Approach Goals in Preparation for Future Learning. Journal of the Learning Sciences, 21(3), 399-432. doi:10.1080/10508406.2011.651232

Broadbent, D. (1958). Perception and communication. Elmsford, NY, US: Pergamon Press. doi. org/10.1037/10037-000.

Clark, R., Kirschner, P. A., \& Sweller, J. (2012). Putting students on the path to learning: The case for fully guided instruction. American Educator. Retrieved from https://www.aft.org/sites/default/files/periodicals/Clark.pdf

D’Mello, S., \& Graesser, A. (2014). Confusion. In P. A. Alexander, R. Pekrun, \& L. Linnenbrink-Garcia (Eds.), International handbook of emotions in education (pp. 289-310). New York, NY: Taylor and Francis.

Di Leo, I., Muis, K. R., Singh, C. A., \& Psaradellis, C. (2019). Curiosity... Confusion? Frustration! The role and sequencing of emotions during mathematics problem solving. Contemporary educational psychology, 58, 121-137. doi:10.1016/j.cedpsych.2019.03.001

Feriver, S., Olgan, R., Teksoz, G., \& Barth, M. (2019). Systems Thinking Skills of Preschool Children in Early Childhood Education Contexts from Turkey and Germany. Sustainability, 11(5), 26. doi:10.3390/su11051478

Kapur, M. (2014). Productive Failure in Learning Math. Cognitive science, 38(5), 1008-1022. doi:10.1111/cogs.12107
Loibl, K., Roll, I., \& Rummel, N. (2017). Towards a Theory of When and How Problem Solving Followed by Instruction Supports Learning. Educational psychology review, 29(4), 693-715. doi:10.1007/s10648-016-9379-х

Loibl, K., \& Rummel, N. (2014). Knowing what you don't know makes failure productive. Learning and Instruction, 34, 74-85. doi:10.1016/j.learninstruc.2014.08.004

Pekrun, R., Vogl, E., Muis, K. R., \& Sinatra, G. M. (2017). Measuring emotions during epistemic activities: the Epistemically-Related Emotion Scales. Cognition and Emotion, 31(6), 1268-1276.

Schwartz, D. L., Chase, C. C., Oppezzo, M. A., \& Chin, D. B. (2011). Practicing Versus Inventing With Contrasting Cases: The Effects of Telling First on Learning and Transfer. Journal of educational psychology, 103(4), 759-775. doi:10.1037/a0025140

Schwartz, D. L., \& Martin, T. (2004). Inventing to prepare for future learning: The hidden efficiency of encouraging original student production in statistics instruction. Cognition and instruction, 22(2), 129-184. doi:10.1207/s1532690xci2202_1

Simpson, A., \& Maltese, A. (2017). "Failure Is a Major Component of Learning Anything": The Role of Failure in the Development of STEM Professionals. Journal of Science Education and Technology, 26(2), 223-237. doi:10.1007/s10956-016-9674-9

*Autor de contacto: Susana Carnero-Sierra, carnerosusana@ uniovi.es
MAGISTER

Vol. 32. Núm. I: (2020). Sección extraordinaria 